\title{
CME: Zertifizierte Fortbildung in der Rehabilitation
}

\section{CME: Certified Professional Development in Rehabilitation}

Autor

F. Schliehe

Bibliografie

DOI http://dx.doi.org/

10.1055/s-0033-1333700

Rehabilitation 2013;

52: 1

(c) Georg Thieme Verlag KG

Stuttgart · New York

ISSN 0034-3536

Korrespondenzadresse

Dr. Ferdinand Schliehe

An der Blankenburg 18

49078 Osnabrück

fam.schliehe@t-online.de

\section{Prof. Dr. Wilfried Mau}

Institut für Rehabilitationsmedizin

Medizinische Fakultät

Martin-Luther-Universität

Halle-Wittenberg

Magdeburger Straße 8

06097 Halle (Saale)

reha@medizin.uni-halle.de
Einen wesentlichen Baustein der Qualitätssicherung in der medizinischen und rehabilitativen Versorgung stellt eine ständige Fortbildung der dort tätigen Berufsgruppen dar. Dies gilt besonders vor dem Hintergrund, dass sich medizinisches und allgemein gesundheitsbezogenes Fachwissen ständig durch technologische Entwicklungen, neue wissenschaftliche Erkenntnisse und praktische Erfahrungen erweitert und verändert. Die Fachgesellschaften haben deshalb für ihre jeweiligen Disziplinen eine Reihe von speziellen Fortbildungskonzepten entwickelt und bieten eine Vielzahl von Veranstaltungen für ihre jeweilige Berufsgruppe an. Speziell für in der Medizin (auch Rehabilitation) tätige Ärzte hat sich das Konzept einer kontinuierlichen berufsbegleitenden Fortbildung etabliert, das unter dem Namen „Continuing Medical Education“ (CME) firmiert. Die Grundlagen dafür wurden 2004 auf dem Deutschen Ärztetag beschlossen, nachdem durch das „Gesetz zur Modernisierung der gesetzlichen Krankenversicherung“ die bis dahin berufsrechtlich geregelte ärztliche Fortbildungspflicht zum 1. Januar 2004 verbindlich eingeführt wurde. Vertragsärzte, Vertragspsychotherapeuten und Krankenhausfachärzte sind verpflichtet, Fortbildungszertifikate bei der zuständigen Ärztekammer durch entsprechende Nachweise (in der Regel 250 Fortbildungspunkte innerhalb von 5 Jahren) zu erwerben. Die Fortbildung soll sich dabei nicht nur auf fachspezifische Kenntnisse beschränken, sondern kann auch fachübergreifende, interdisziplinäre, methodische und andere Kenntnisse und Fertigkeiten umfassen. Zertifikate können durch sehr unterschiedliche Veranstaltungsformen und Inhalte erworben werden, auch durch ein mediengestütztes Selbststudium.

Die Herausgeber und der Thieme-Verlag haben beschlossen, ab dieser Ausgabe in regelmäßigen Abständen eine primär für Ärzte und Psychotherapeuten konzipierte zertifizierte Fortbildung im Selbststudium durch besondere Fachartikel anzubieten. Die Beiträge enthalten viele Informationen, die auch für andere Berufsgruppen der multiprofessionellen Kooperation relevant sind. Sie unterliegen einem Reviewverfahren und sind durch die Ärztekammer Nordrhein zertifiziert. Die Zeitschrift „Die Rehabilitation“ wird damit Bestandteil des Fortbildungsportals der Thieme-Fachzeitschriften (vgl. CME.thieme.de), auf dem auch nähere Informationen zu CME sowie zum Verfahren des Zertifikatserwerbs zu erhalten sind.

Verantwortlich für die Fortbildungsbeiträge in dieser Zeitschrift ist Prof. Wilfried Mau, Medizinische Fakultät der Martin-Luther-Universität Halle-Wittenberg, Institut für Rehabilitationsmedizin. Wilfried Mau ist Vizepräsident der
Deutschen Gesellschaft für Rehabilitationswissenschaften (DGRW) und zugleich Sprecher der Kommission für Aus-, Fort- und Weiterbildung der DGRW. Er übernimmt die Koordination der neuen Rubrik „Fort- und Weiterbildung“. An ihn können sich auch Interessenten wenden, die Fachbeiträge für die Fortbildung erstellen möchten. Die Auswertung und Erfolgskontrolle der Multiple-Choice-Fragen zu den Fachbeiträgen übernimmt der Thieme-Verlag unter Beachtung des Datenschutzes.

Der erste CME-Beitrag von Mau und EhlebrachtKönig behandelt die Rehabilitation bei axialen Spondyloarthritiden und zeigt neben einer differenzierten Diagnostik und interdisziplinären Therapie auch die Bedeutung für das ärztlich koordinierte Behandlungsteam auf.

Neben diesem Fachbeitrag umfasst die Ausgabe 7 Artikel zu unterschiedlichen Themen.

Jahed, Bengel und Baumeister untersuchen das Inanspruchnahmeverhalten bei Patienten mit psychischen Belastungen in der somatischen Rehabilitation. Insgesamt weisen psychisch belastete Patienten eine hohe Bereitschaft für psychotherapeutische Behandlungen auf. Diese Bereitschaft setzt sich jedoch post-rehabilitativ nicht um, weshalb eine gezieltere Motivationsförderung in der stationären Phase notwendig erscheint. Der Beitrag von Neuderth et al. stellt ein standardisiertes Qualitätssicherungsprogramm für Einrichtungen der stationären Vorsorge für Mütter und Väter vor, das bundesweit einsetzbar ist. In dem anschließenden Beitrag behandeln Frank et al. die Ergebnisse aus einem Pilotprojekt zum Management von Trachealkanülen im Rahmen der neurologischen Rehabilitation. Es werden u.a. Kriterien vorgestellt, anhand derer über eine Dekanülisierung entschieden werden kann. Hetzel und Schmidt erarbeiten anhand von Arbeitsmarktindikatoren eine empirische Regionalklassifikation zur Anwendung in der beruflichen Rehabilitation am Beispiel des Teilarbeitsmarktes für Bürofachkräfte. Im nachfolgenden Beitrag berichten Nagl, Ullrich und Farin die Ergebnisse einer qualitativen Studie über die Verständlichkeit von Patientenschulungen in der orthopädischen Rehabilitation und zeigen Verbesserungspotenzial auf. Abschließend aktualisieren Schliehe und Ewert eine Bestandsaufnahme zur ICF von 2006 im Auftrag der Deutschen Gesellschaft für Rehabilitationswissenschaften. Trotz breiter Akzeptanz stößt die Nutzung der ICF noch an deutliche Grenzen. 